\title{
Do we decrease the frequency of paralytic ileus after colorectal surgery using an ERAS protocol?
}

D. Carballo, S. Madueño Gonzalez, B. San Antonio San Román, M. Barbero Mielgo, A. Herrero Cano, J. García Fernández; Department of Anesthesiology and Perioperative Medicine Hospital Puerta de Hierro - Madrid (Spain).

\section{Goals:}

Surgery is the choice treatment and the only curative option for many colorectal diseases. The surgery constitutes an agression with hormonal and metabolic alterations, as well as a proinflammatory systemic response. One of the associated postoperative complications is the alteration of gastrointestinal motility in form of postoperative paralytic ileus (PPI).

Several mechanisms have been suggested as PPI causatives. Most of these factors increase the inflammatory response and edema, reducing intestinal motility.

Through a series of perioperative measures and a multimodal approach of the patient, ERAS protocols aim to reduce the alteration of the homeostasis that surgery implies, and have been shown to reduce morbidity and mortality.

Our goal is to check if there is lower PPI frequency after ERAS protocol measures application, compared to the standard cares, prior to its establishment. Our second goal is to analize if the decrease in the ileus frequency, as well as other complications, may shorten hospital stay.

\section{Materials and Methods:}

We compared a retrospective Pre-ERAS group and a prospective ERAS group:

1. ERAS: 165 patients between january and november 2017 under ERAS protocol measures.

\begin{tabular}{|c|c|c|}
\hline \multirow{4}{*}{ 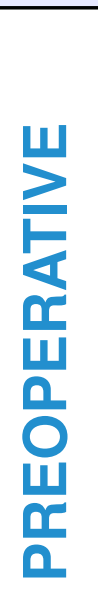 } & 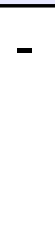 & $\begin{array}{l}\text { Patient and relatives empowerment. More } \\
\text { information and involvement in the recovery } \\
\text { process }\end{array}$ \\
\hline & - & $\begin{array}{l}\text { Nutritional optimization. Patient blood } \\
\text { management. }\end{array}$ \\
\hline & - & Carbohydrate drinks the night before surgery. \\
\hline & - & of analgesic coadjuvants. \\
\hline \multirow{6}{*}{ 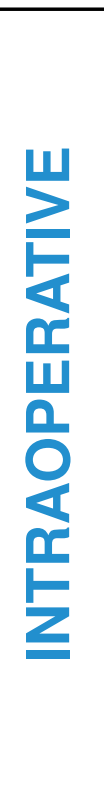 } & & Preferred laparoscopic approach. \\
\hline & & Use of IV lidocaine and MG sulphate. \\
\hline & & Restrictive fluid administration (3-5 ml/kh/h) \\
\hline & & $\begin{array}{l}\text { Pulmonary protective ventilation, recruitment } \\
\text { maneuvers, } \mathrm{FiO} 2>0.6\end{array}$ \\
\hline & - & Avoid nasogastric tube rutinary use. \\
\hline & & $\begin{array}{l}\text { Thoracic epidural, neuromuscular blockade } \\
\text { and NSAIDs use to decrease opioids } \\
\text { administration. }\end{array}$ \\
\hline \multirow{5}{*}{ 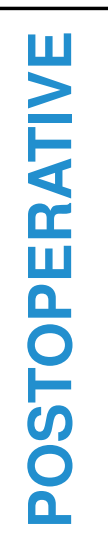 } & & $\begin{array}{l}\text { Avoid IV fluid administration in the immediate } \\
\text { postoperative period. }\end{array}$ \\
\hline & & Early onset of oral intake. \\
\hline & & IV Iron therapy administration \\
\hline & & Early mobilization. \\
\hline & & Postoperative chewing gum administ \\
\hline
\end{tabular}

2. Pre-ERAS: 65 patients between july and october 2015 , under standard measurements.

\section{Results:}

There were no statistically significant differences between the two groups in terms of sex, age, laparoscopic or open approach or mean duration of surgery; There were differences in postoperatory opioids use:

\begin{tabular}{|c|c|c|c|}
\cline { 2 - 4 } \multicolumn{1}{c|}{} & ERAS & $\begin{array}{c}\text { PRE- } \\
\text { ERAS }\end{array}$ & $\mathrm{p}$ \\
\hline SEX & Male $61 \%$ & Male $62 \%$ & $\mathrm{p}=0.85$ \\
\hline MEAN AGE $(\mathrm{y})$ & 67,63 & 64,8 & $\mathrm{p}=0.19$ \\
\hline $\begin{array}{c}\text { LAPAROSCOPIC } \\
\text { APPROACH }\end{array}$ & $65 \%$ & $58 \%$ & $\mathrm{p}=0.43$ \\
\hline $\begin{array}{c}\text { MEAN SURGERY } \\
\text { DURATION (mins) }\end{array}$ & 195,6 & 76,12 & $\mathrm{p}=0.18$ \\
\hline $\begin{array}{c}\text { PUOSTOP } \\
\text { OPIOIDS USE }\end{array}$ & $35,7 \%$ & $80 \%$ & $\mathrm{p}=0.001$ \\
\hline
\end{tabular}

We found statistically significant differences in terms of lower ileus frequency in ERAS group, $16,88 \%$ vs $29,23 \%$ in pre ERAS group $(p=0.039)$

We also found less hospitalary lenght of stay: A median lenght of stay of 8,89 days (sd 7,0$)$ vs 11,91 (sd 10,9) in pre-ERAS group $(p=0,049)$

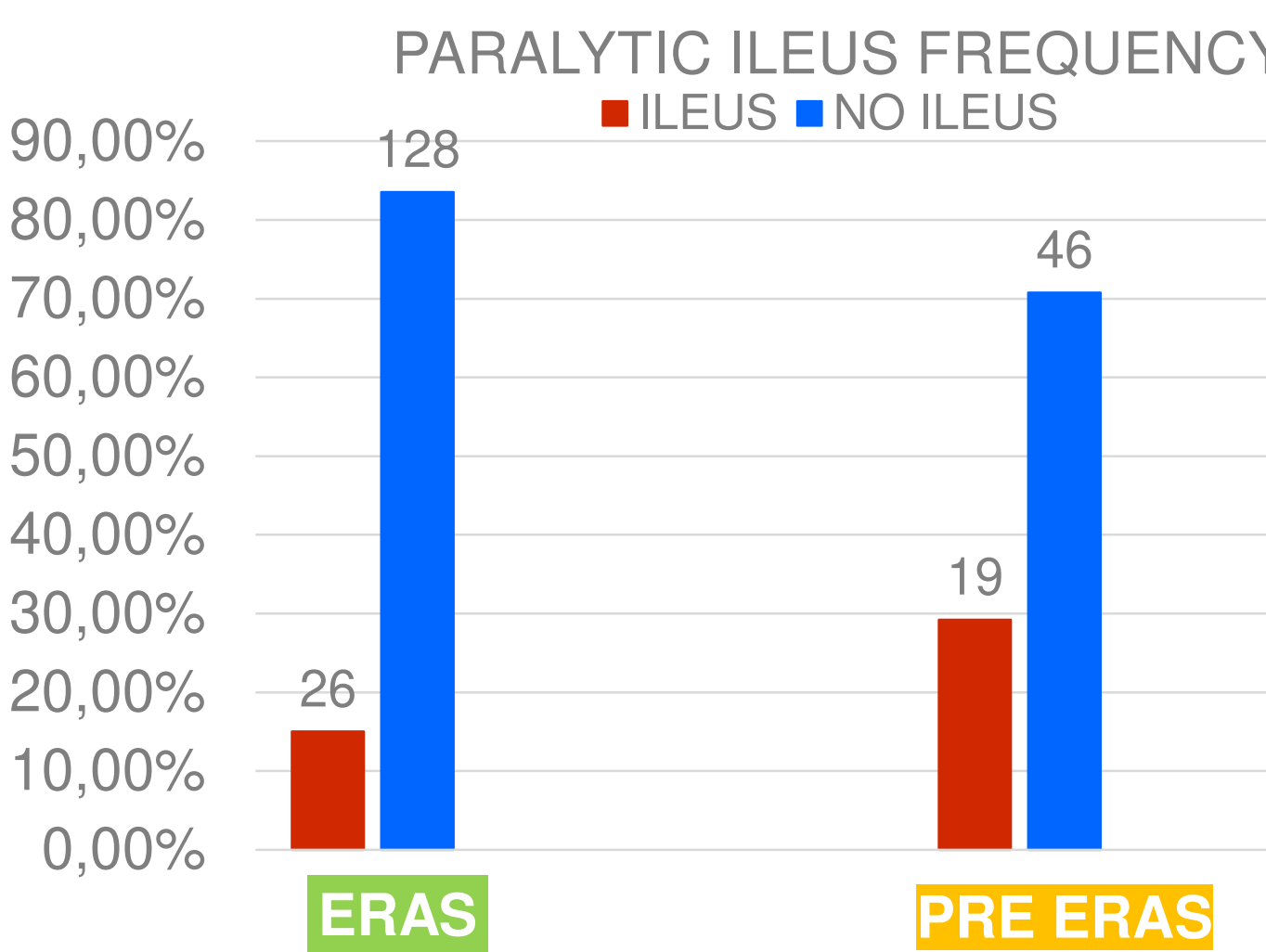

Conclusions:

Our results show that the application of an ERAS protocol seems to be related with a lower frequency of PPI and may allow an early hospital discharge and a reduction in health expenditure, however, more evidence should be obtained trough future research. 


\begin{tabular}{|c|c|c|c|c|c|}
\cline { 2 - 6 } \multicolumn{1}{c|}{} & SEX & MEAN AGE (Y) & LAPAROSCOPIC & $\begin{array}{c}\text { MEAN SURGERY } \\
\text { APURATION } \\
\text { (mins) }\end{array}$ & PPIOSTOP USE \\
\hline ERAS & Male 61\% & 67,63 & $65 \%$ & 195,6 & $35,7 \%$ \\
\hline Pre-ERAS & Male 62\% & 64,8 & $58 \%$ & 76,12 & $80 \%$ \\
\hline SIGNIFICANCE & $\mathrm{p}=0.85$ & $\mathrm{p}=0.19$ & $\mathrm{p}=0.43$ & $\mathrm{p}=0.18$ & $\mathrm{P}=0.001$ \\
\hline
\end{tabular}

\begin{tabular}{|c|c|c|c|}
\cline { 2 - 4 } \multicolumn{1}{c|}{} & ERAS & PRE-ERAS & SIGNIFICANCE \\
\hline SEX & Male 61\% & Male 62\% & $\mathrm{p}=0.85$ \\
\hline MEAN AGE (y) & 67,63 & 64,8 & $\mathrm{p}=0.19$ \\
\hline $\begin{array}{c}\text { LAPAROSCOPIC APPROACH } \\
\text { MEAN SURGERY DURATION } \\
\text { (mins) }\end{array}$ & $65 \%$ & $58 \%$ & $\mathrm{p}=0.43$ \\
\hline $\begin{array}{c}\text { POSTOP OPIOIDS USE } \\
\text { MOS }\end{array}$ & $35,7 \%$ & $80 \%$ & $\mathrm{p}=0.001$ \\
\hline
\end{tabular}

